\title{
Unique features of myogenesis in Egyptian cobra (Naja haje) (Squamata: Serpentes: Elapidae)
}

\author{
Eraqi R. Khannoon ${ }^{1} \cdot$ Weronika Rupik ${ }^{2}$. \\ Damian Lewandowski $^{3}$. Magda Dubińska-Magiera ${ }^{3}$. \\ Elwira Swadźba $^{2} \cdot$ Małgorzata Daczewska $^{3}$
}

Received: 25 February 2015 / Accepted: 21 May 2015 / Published online: 30 May 2015

(C) The Author(s) 2015. This article is published with open access at Springerlink.com

\begin{abstract}
During early stages of myotomal myogenesis, the myotome of Egyptian cobra (Naja haje) is composed of homogenous populations of mononucleated primary myotubes. At later developmental phase, primary myotubes are accompanied by closely adhering mononucleated cells. Based on localization and morphology, we assume that mononucleated cells share features with satellite cells involved in muscle growth. An indirect morphological evidence of the fusion of mononucleated cells with myotubes is the presence of numerous vesicles in the subsarcolemmal region of myotubes adjacent to mononucleated cell. As differentiation proceeded, secondary muscle fibres appeared with considerably smaller diameter as compared to primary muscle fibre. Studies on $N$. haje myotomal myogenesis revealed some unique features of muscle differentiation. TEM analysis showed in the $N$. haje myotomes two classes of muscle fibres. The first class was characterized by typical for fast muscle fibres regular distribution of myofibrils which fill the whole volume of muscle fibre sarcoplasm. White muscle fibres in studied species were a prominent group of muscles in the myotome. The second class showed tightly paced myofibrils surrounding the centrally located nucleus accompanied by numerous vesicles of different
\end{abstract}

Handling Editor: Pavel Dráber

Małgorzata Daczewska

malgorzata.daczewska@uni.wroc.pl

1 Zoology Department, Faculty of Science, Fayoum University, Fayoum 63514, Egypt

2 Department of Animal Histology and Embryology, University of Silesia, 9 Bankowa Str., 40-007 Katowice, Poland

3 Department of Animal Developmental Biology, Institute of Experimental Biology, University of Wrocław, 21 Sienkiewicza Str., 53-335 Wrocław, Poland diameter. The sarcoplasm of these cells was characterized by numerous lipid droplets. Based on morphological features, we believe that muscle capable of lipid storage belong to slow muscle fibres and the presence of lipid droplets in the sarcoplasm of these muscles during myogenesis might be a crucial adaptive mechanisms for subsequent hibernation in adults. This phenomenon was, for the first time, described in studies on $N$. haje myogenesis.

Keywords Myogenesis $\cdot$ Satellite cells $\cdot$ TEM $\cdot$ Naja haje . Snake

\section{Introduction}

Differentiation of the trunk (myotomal) muscles has been investigated in detail in different groups of vertebrates including fishes, amphibians, birds and mammals. The myotomal muscles in all studied vertebrate specimens originate from unsegmented paraxial mesoderm which, during subsequent stages of embryonal development, is subdivided into repetitive cell units called somites. The somites then differentiate into three compartments: the dermomyotome with a well-developed dorsomedial lip and ventrolateral lip, the sclerotome and afterwards into the myotome. The main part of the myotome is occupied by trunk muscles which differentiate in situ (reviewed by Bentzinger et al. 2012). Studies on development of amniote (birds and mammals) trunk muscles have revealed that the dermomyotome is the main source of trunk muscle progenitor cells, which express the paired-box transcription factors Pax 3 and Pax7. It has been shown that Pax 3 is required to establish the myogenic potential of differentiating cells, whereas Pax 7 is required for the specification and maintenance of satellite cells during myogenesis (Horst et al. 2006; 
Buckingham and Realaix 2007; Kacperczyk et al. 2009; Seale et al. 2000; Olguin and Olwin 2004; Zammit et al. 2006).

During the vertebrates' development, myogenic progenitors differentiate into myoblasts, which differentiate into myotubes, a developing immature skeletal muscle fibres with a centrally located nucleus in the sarcoplasm. The final stage of myogenesis is a formation of mature muscle fibres - cylindrical multinucleate cells with peripherally located nuclei. Muscles grow through two mechanisms: hypertrophy and hyperplasia. During hypertrophy, the fibre size increases due to the addition of new nuclei into sarcoplasm of pre-existing fibres, while hyperplasia is a process of new muscle fibre formation. It is well evidenced that in amniotes, Pax 7 muscle progenitor cells participate in both processes in the prenatal stage. It is noteworthy that, after birth, a number of muscle fibres remain unchanged in mammals and birds (Rowe and Goldspink 1969; Fowler et al. 1980; Nimmo and Snow 1983; Brown 1987; Rosenblatt and Woods 1992; Schadereit et al. 1995). Postnatal growth is mainly due to hypertrophy, although some reports have revealed an increase in fibre number shortly after birth (Swatland 1975; Rehfeldt and Fiedler 1984; Summers and Medrano 1994; Fiedler et al. 1998).

Among all vertebrates, reptile myogenesis and myotomal muscle growth are poorly known. Studies on reptile myogenesis have been conducted on several species (Chinese soft-shelled turtle Pelodiscus sinensis and sand lizard Lacerta agilis); among them, only sand lizard studies on myogenesis shed light on the origin of muscle progenitor cells and growth of myotomal muscles (Nagashima et al. 2005; Rupik et al. 2012). It was found that in the sand lizard, similarly to birds and mammals, the dermomyotome is the main source of muscle Pax3-positive progenitor cells. Studies conducted by Rupik et al. (2012) revealed that muscle growth in the sand lizard is due to Pax3/Pax7-positive mononucleated cells. In comparison to the sand lizard, snakes, e.g. Naja haje, represent a different mode of locomotion. In our studies, we attempted to answer the question whether mode of locomotion influences the pattern of muscle differentiation and growth. In the present study, we investigated trunk muscle differentiation and growth in the previously unstudied Egyptian cobra (N. haje) in order to demonstrate whether myogenesis in the studied species displays a unique character, not observed in other vertebrates.

\section{Material and methods}

The Egyptian cobra (N. haje L. (1758): Elapidae, Reptilia) is one of the largest cobra species native to Africa. It is terrestrial and crepuscular or nocturnal. Adult female cobras attain sexual maturity after 2 to 3 years. They lay 17 to 22 eggs in a clutch. Oviposition occurs 60-100 days after copulation in early summer (Schleich et al. 1996). Fertilized female
Egyptian cobras for this study were collected from the Nile Delta region of Egypt in June 2012-2013. The animals were kept in vivaria in an open farm area, in conditions similar to those in the wild, until the eggs were laid, and then they were released into their native area. All specimens used in the experiment were captured according to the Egyptian regulations concerning the protection of wild species (Convention on Biological Diversity ratified in 1992 and 1994). The Egyptian cobra is not included in the Washington Convention of 1973. The eggs of Egyptian cobra $(n=100)$ after oviposition were carefully collected and brought to the laboratory of the Zoology Department, Faculty of Science, Fayoum University, where they were placed in plastic boxes filled with moistened perlite (at $85-90 \%$ moisture) and with ventilation holes. The eggs were incubated at $30{ }^{\circ} \mathrm{C}$, reflecting ambient seasonal temperatures in the wild. Embryos used for investigation were isolated at regular intervals from egg laying to hatching. Embryonic development at $30^{\circ} \mathrm{C}$ in laboratory conditions from when the eggs are laid until they hatch lasts 51-54 days (Khannoon and Evans 2014). The age of the embryos was calculated using the table for $N$. haje development (Khannoon and Evans 2014). The study of myotomal muscle differentiation and growth of $N$. haje included four developmental stages from stages 3 to 6 .

For light and electron microscopic techniques, small pieces of embryonic body wall including differentiating muscle tissue were fixed in a 1:1 mixture of $2.5 \%$ glutaraldehyde (Sigma-Aldrich) and $2.0 \%$ paraformaldehyde (Sigma-Aldrich, St. Louis, MO, USA) in $0.1 \mathrm{~m}$ phosphate buffer, $\mathrm{pH} 7.4$, for $24 \mathrm{~h}$ at $4{ }^{\circ} \mathrm{C}$. The material was repeatedly rinsed in the same buffer and post-fixed for $2 \mathrm{~h}$ in $1 \% \mathrm{OsO}_{4}$ (SigmaAldrich) in $0.1 \mathrm{M}$ phosphate buffer. Following rinsing in phosphate buffer, the material was dehydrated first in a graded alcohol series and then in acetone and embedded in epoxy resin Epon 812 (Sigma-Aldrich) (Luft 1961). This procedure of fixation appears to be the best for different embryonic reptilian tissues (Rupik 2002, 2011, 2012, 2013; Swadźba and Rupik 2010, 2012). The Epon blocks were cut on Leica Ultracut UCT (Leica, Wetzlar, Germany) and Reichert Ultracut E ultramicrotome (Leica, Wetzlar, Germany). Semithin sections $(0.6 \mu \mathrm{m})$ were collected on glass slides and stained with methylene blue in $1 \%$ borax solution (SigmaAldrich) and examined under a light microscope Olympus BX60 and Olympus BHS light microscopes (Olympus Corp., Tokyo, Japan). Ultrathin sections were collected on 200 mesh copper grids, stained with uranyl acetate and lead citrate according to the standard protocol (Reynolds 1963) and examined in a Hitachi H500 (Hitachi Ltd., Tokyo, Japan; 75 kV) and Zeiss EM 900 (Carl Zeiss AG, Oberkochen, Germany; $80 \mathrm{kV}$ ) transmission electron microscopes. Histological material was processed and analysed in the Department of Animal Histology and Embryology, University of Silesia, and in the Department of Animal Developmental Biology, Institute of Experimental Biology, University of Wrocław. 


\section{Results}

\section{Myotomal muscles differentiation and growth in $N$. haje}

Myotomal muscle formation of $N$. haje was analysed in the light microscope and TEM. At stage 3, the myotome of $N$. haje is composed of homogeneous populations of mononucleated primary myotubes containing centrally located homogeneous nuclei (Fig. 1a). Sarcoplasm of these cells revealed the presence of a few myofibrils arranged in an irregular way, numerous mitochondria, Golgi apparatus and glycogen granules. At this stage of development, neighbouring myotubes are closely attached to each other (Fig. 1b). At later developmental phase (stage 4), light microscope analysis revealed that primary myotubes are accompanied by closely adhering mononucleated cell (Fig. 1c). As differentiation proceeded (stage 5), secondary muscle fibres appear. The secondary muscle fibres are distinguished by considerably smaller diameter as compared to primary muscle fibre (Fig. 1d). At this stage of myogenesis, numbers of myofibrils in sarcoplasm of myotubes were higher (Fig. 1e). Ultrastructural analysis showed that mononucleated cells closely adhering to myotubes are composed of a prominent nucleus with heterochromatin located mainly under the nuclear envelope. The nucleus is surrounded by a narrow rim of cytoplasm devoid of glycogen granules and elements of contractile apparatus (Fig. 1f). Localization of mononucleated cells suggests their potential involvement in muscle growth due to their fusion with myotubes. This is plausible since the detailed ultrastructural analysis showed the presence of numerous vesicles in the subsarcolemmal region of myotubes adjacent to mononucleated cells. At this developmental stage, as a result of progressing myogenic cell fusion, elongated, multinucleated myotubes appear (Fig. 1g).

\section{Unique features of muscle differentiation in $N$. haje}

During myotomal myogenesis (stage 5), developing muscle fibres of $N$. haje do not form a homogeneous population. Undertaken studies revealed the presence of two classes of muscle fibres distinguished by the distribution of myofibrils. Class I is characterized by typical regular distribution of myofibrils which fill the whole volume of the muscle fibre sarcoplasm (Fig. 2a). Class II features tightly packed myofibrils surrounding a centrally located nucleus accompanied by numerous vesicles. They are localized only in the proximity of the nucleus and do not occur in the peripheral region of the sarcoplasm, which is filled with numerous mitochondria and endoplasmic reticulum. Sarcoplasm of II class of muscle fibres contains numerous glycogen granules among myofibrils and in the peripheral region (Fig. 2b, c). The nuclei of class II muscle fibres are rich in heterochromatin localized beneath the nuclear envelope and in the internal part of the nucleus
(Fig. 2d). Sarcoplasm of these cells is also characterized by numerous lipid droplets surrounded by glycogen granules (Fig. 2e, f).

\section{Discussion}

\section{Differentiation and growth of muscles in N. haje}

Skeletal muscle differentiation and growth in vertebrates have been investigated in detail in model species (zebrafish, Xenopus laevis, chick, mouse) (reviewed by Bentzinger et al. 2012; Kiełbówna and Jędrzejowska 2012; Rossi and Messina 2014). These model vertebrate species provided detailed information at the morphological and the molecular level of muscle development in this phylum. Studies on reptilian developmental biology including myogenesis are difficult and rarely attempted (Nagashima et al. 2005; Rupik et al. 2012; Kusumi et al. 2013). To better understand the mechanisms of trunk muscle differentiation and growth in reptiles, we chose the Egyptian cobra. Our results revealed that in the studied species during early steps of myogenesis, the myotome was composed of a homogeneous population of myogenic cells consisting of mononucleated primary myotubes. The structure and morphology of these cells in $N$. haje myotomes resemble mononucleated myocytes observed in primary myotomes in birds and mammals and also in the sand lizard, a representative of reptilians which, in comparison to Egyptian cobra, represents a different mode of locomotion. During subsequent stages of muscle fibres differentiation, myotubes were accompanied by mononucleated cells. Based on localization and morphology, these cells share features with satellite cells, described in detail in many vertebrates (fish, amphibians, birds and mammals) (Zammit et al. 2006; Kacperczyk et al. 2009, 2011; Daughters et al. 2011; reviewed by Siegiel et al. 2013; Yin et al. 2013). TEM analysis revealed that satellite cells in the studied species are spindle-shaped composed of a prominent nucleus with heterochromatin located beneath the nuclear envelope. The narrow rim of their cytoplasm is devoid of glycogen granules and elements of contractile apparatus. Compared to differentiating myotubes, the ultrastructure of satellite cells showed an undifferentiated state. In the sand lizard, similarly to birds and mammals, satellite cells that adjoin the differentiating muscle fibres express Pax 7 protein (Rupik et al. 2012). The origin of these cells in N. haje has not been studied. Many different lines of evidence indicate that in amniotes, mononucleated cells that adjoin the myotubes and/or muscle fibres originate from the dermomyotome. Studies on sand lizard myogenesis revealed that, as in other vertebrates, these cells are myogenic precursors involved in muscle growth (both hypertrophic and hyperplastic) (Rupik et al. 2012). Increase in the number of nuclei in myotubes (hypertrophy) in subsequent stages of myogenesis 

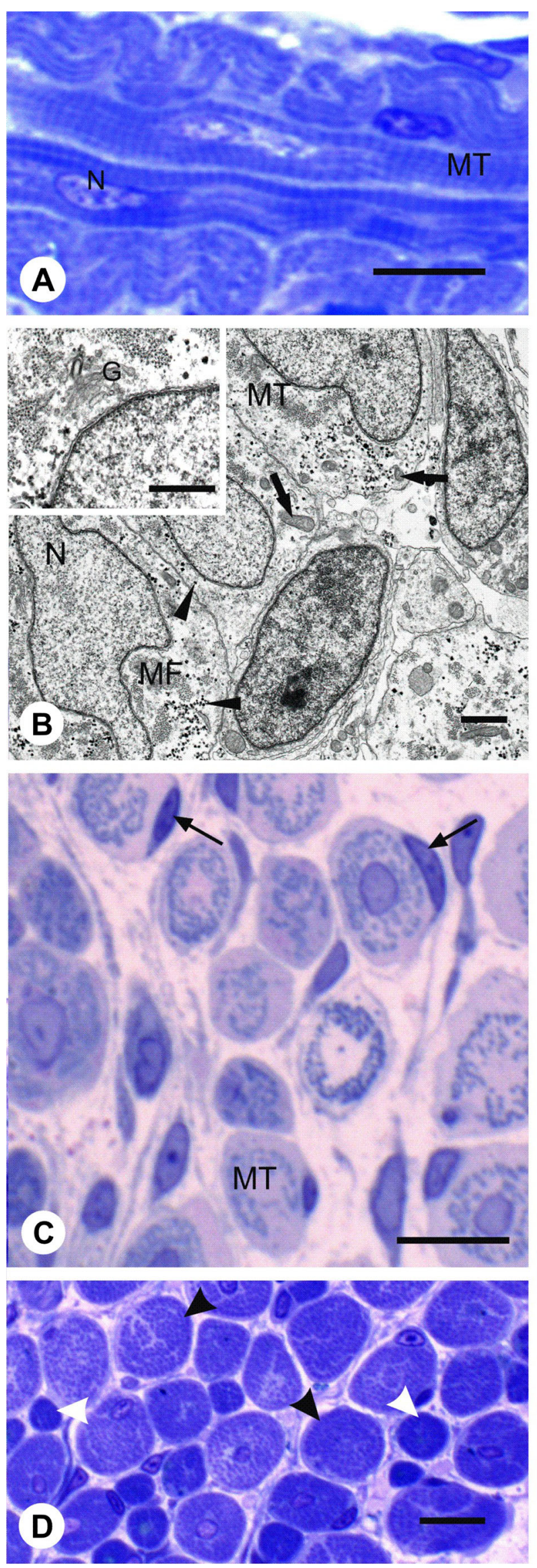
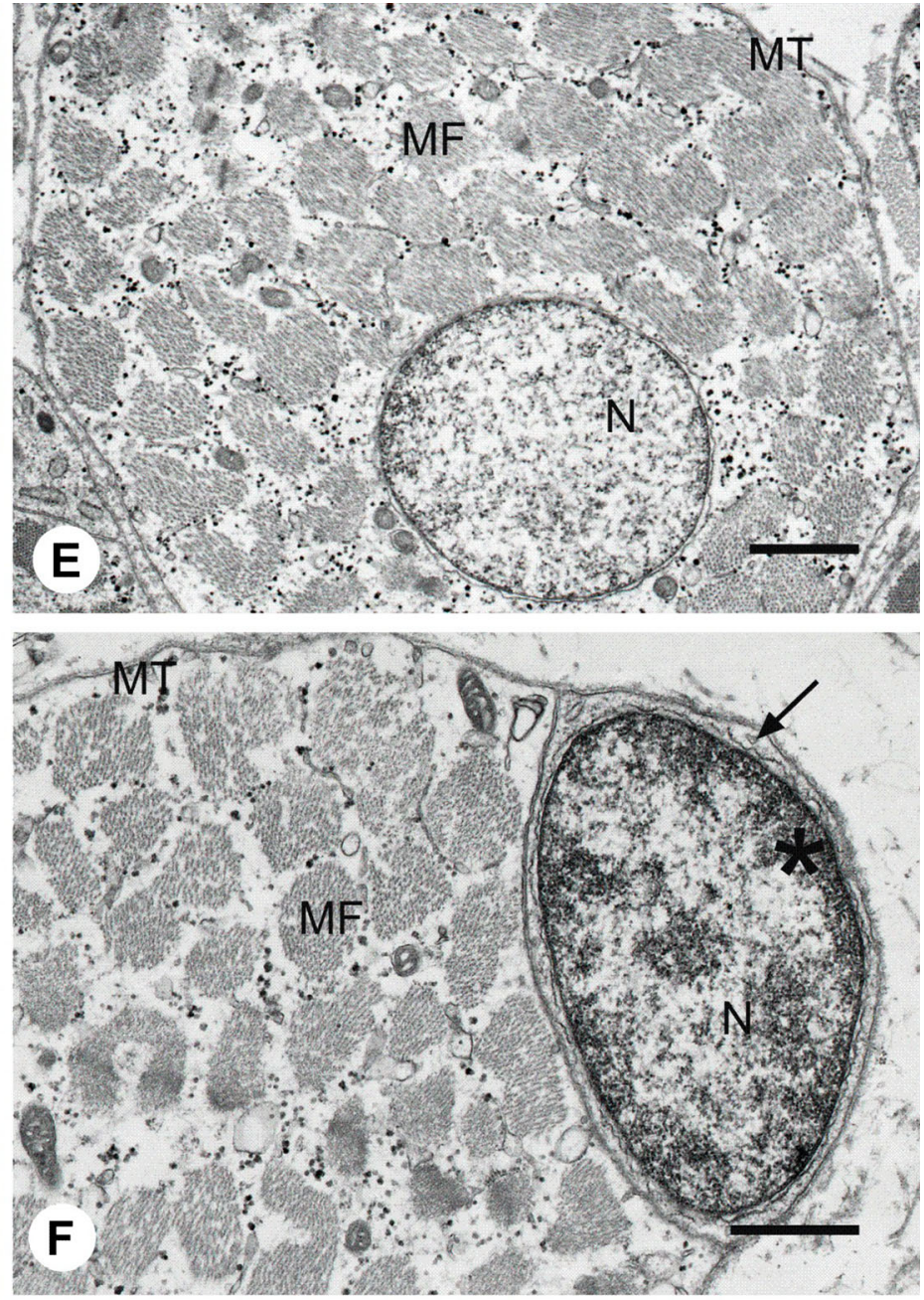

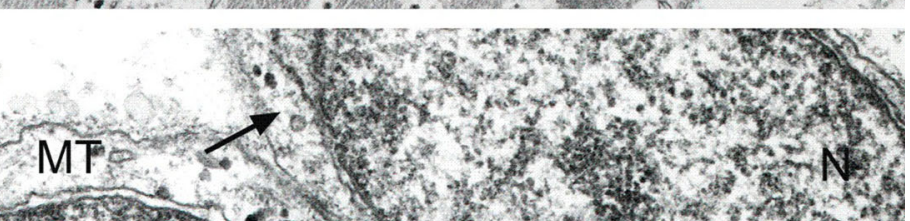

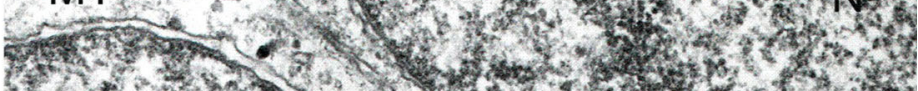
K.

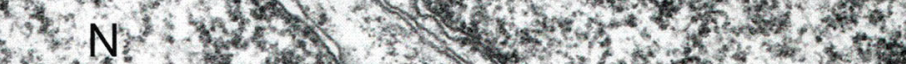

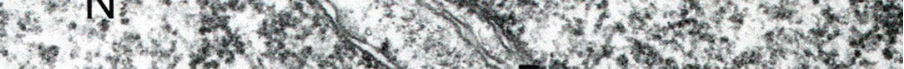

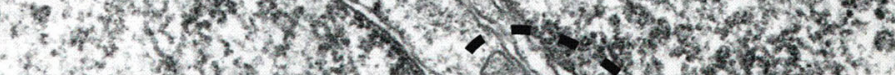

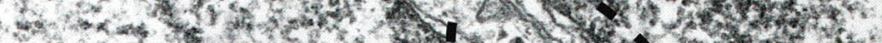

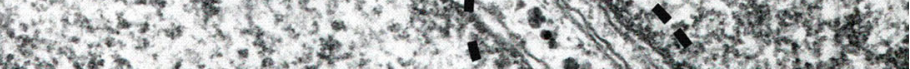

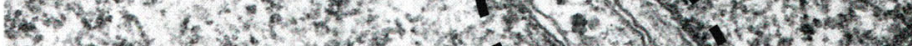

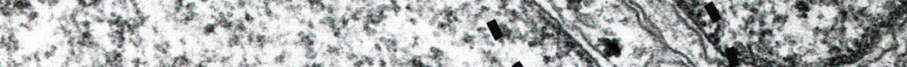

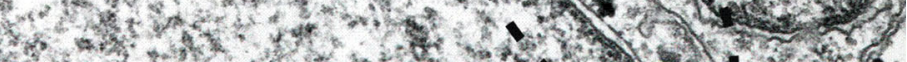
V.

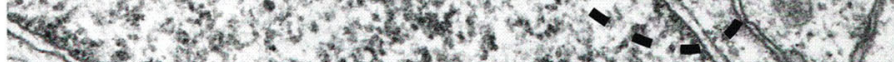

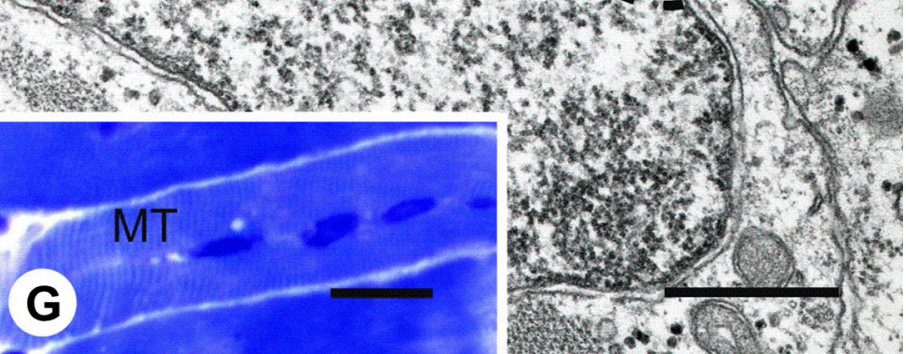


4 Fig. 1 Myotomal muscles differentiation and growth in N. haje. a Stage 3: Longitudinal section of the posterior trunk myotome. Mononucleated myotubes $(M T)$ centrally located homogenous nucleus $(N)$. Semi-thin section, methylene blue staining, scale bar: $10 \mu \mathrm{m}$. b Stage 3: Ultrastructure of the posterior trunk myotome. Myotubes (MT), myofibril $(M F)$, nucleus $(N)$, glycogen (black arrowhead), mitochondria (black arrows). Inset: Golgi apparatus $(G)$. TEM, scale bar: $1 \mu \mathrm{m}$. c Stage 4: Cross-sections of the posterior trunk myotome. Primary myotube (MT), mononucleated cells (black arrows). Semi-thin section, methylene blue staining, scale bar: $10 \mu \mathrm{m}$. d Stage 5: Crosssections through the posterior trunk myotome. Primary myotubes (black arrowheads), secondary myotubes (white arrowheads). Semi-thin section, methylene blue staining, scale bar: $10 \mu \mathrm{m}$. e Stage 5: Crosssections through the posterior trunk myotome. Ultrastructure of myotubes $(M T)$, myofibrils $(M F)$, nucleus $(N)$. TEM, scale bar: $1 \mu \mathrm{m}$. f Stage 5: Cross-sections through the posterior trunk myotome. Myotube $(M T)$, myofibril $(M F)$, mononucleated cell (black arrow), nucleus $(N)$, heterochromatin (asterisk) under the nuclear envelope. TEM, scale bar: $1 \mu \mathrm{m}$. g Stage 5: Cross-sections through the posterior trunk myotome. Mononucleated cell (black arrow), myotube (MT), vesicles in the subsarcolemmal sarcoplasm of the myotubes plasma membranes (circled), myotube nucleus $(N)$ nucleus of mononucleated cell $(N)$. Inset: multinucleated myotube (MT). TEM, scale bar: $1 \mu \mathrm{m}$

and occurrence of new muscle fibres (hyperplasia) with smaller diameter compared to the primary muscle fibres is indirect evidence of participation of these cells in $N$. haje muscle growth. The presence of numerous vesicles budding from the myotube sarcolemma, closely adhering to the surface of the myotubes, is morphological evidence of the fusion of these cells in N. haje. Similar vesicles have also been found below the plasmalemma of fusing chicken myoblasts in vitro and in vivo during teleost muscle growth. During muscle growth in the sand lizard, detailed TEM analysis also showed numerous vesicles in the subsarcolemmal sarcoplasm of muscle fibres and the partial fusion of muscle fibre and mononucleated cell plasma membranes (Kalderon and Gilula 1979; Merkel 1995; Daczewska 2001; Kacperczyk and Daczewska 2006, 2008; Rupik et al. 2012). Data obtained from these studies strongly support the hypothesis that mononucleated cells/ satellite cells in $N$. haje participate in muscle growth. Our studies revealed that the pattern of muscle differentiation and growth in reptiles is independent of the mode of locomotion (see lizards versus snakes).

Locomotor adaptations are essential for survival as locomotion plays a crucial role in many biological functions including the capturing of prey, competing with possible rivals and escaping predators (Garland and Losos 1994; Irshick and Garland 2001; Aubret 2004). Locomotion strongly differs from that of four-legged taxa whose propulsion and support are mainly ensured by the limbs (Aerts et al. 2000; McElroy and Reilly 2009). Indeed, snakes can use their entire body to generate propulsion when in contact with the substrate. The axial muscles of snakes are notable for having long tendons within individual segments that span several vertebrae. Consequently, muscles that extend anteriorly have a constraint on their length as their origins locate closer to the skull.
Compared to other amniotic vertebrates, snakes have extraordinarily long axial muscles in which the contractile tissue commonly spans 3-6 vertebrae, and tendons may span from one to more than 30 vertebrae, depending on the particular muscle and species (Mosauer 1935; Gasc 1981; Jayne 1982;). In lizards, axial muscles stabilize the trunk during locomotion, and according to Ritter (1996), this stabilizing role is a basal feature of lizards. Enzymatic and histochemical analysis revealed that lizards possess both slow and fast muscles (proportion of muscle types depends on locomotor behaviour) whereas white muscle fibres in snakes represent a prominent group of muscles in myotomes (Gleeson et al. 1980; Guthe 1981; Moritz and Schilling 2013). Data obtained from studies on muscle differentiation and growth in $N$. haje provide new evidence confirming conservation of the myogenic programme in amniotes. Further research on reptile myotomal myogenesis should focus on the red and white muscle differentiation to show differences among muscle fibres precursors that will give a rise the adult muscle fibres.

\section{Unique features of muscle differentiation in $N$. haje}

Although muscle differentiation and growth in $N$. haje share great many similarities to myogenesis described in amniotes (in birds, mammals and sand lizard), our studies revealed some unique features of muscle differentiation in the studied species. As development proceeded, light microscope and TEM analysis revealed in the $N$. haje myotomes two classes of muscle fibres. The first class was characterized by typical for white/fast muscle fibres regular distribution of myofibrils which fill the whole volume of the muscle fibre sarcoplasm. White muscle fibres in studied species are a prominent group of muscles in the myotome. It has been reported that majority of fibres in snake body musculature are fast muscle fibres that are important for quicker movements and for shortening to do work (Guthe 1981). The second class showed tightly packed myofibrils surrounding the centrally located nucleus accompanied by numerous vesicles of different diameter. We suppose class I muscle fibres originate from primary and secondary myotubes, whereas class II muscle fibres may originate only from primary myotubes. This assumption was based on morphological observation regarding diameter of myotubes and muscle fibres (Kacperczyk et al. 2011). It is commonly accepted that in vertebrates, during myogenesis, secondary muscle fibres have smaller diameter compared to the primary muscle fibres. A characteristic feature of the second class of fibres is the presence of peripheral sarcoplasm containing no contractile apparatus. The sarcoplasm of these cells is also characterized by numerous lipid droplets. This phenomenon was, for the first time, described in our studies on $N$. haje myogenesis. It is commonly accepted that reptiles, as ectothermic animals, undergo hibernation as an adaptation to different habitats. Hibernation occurs also during the $N$. haje annual 

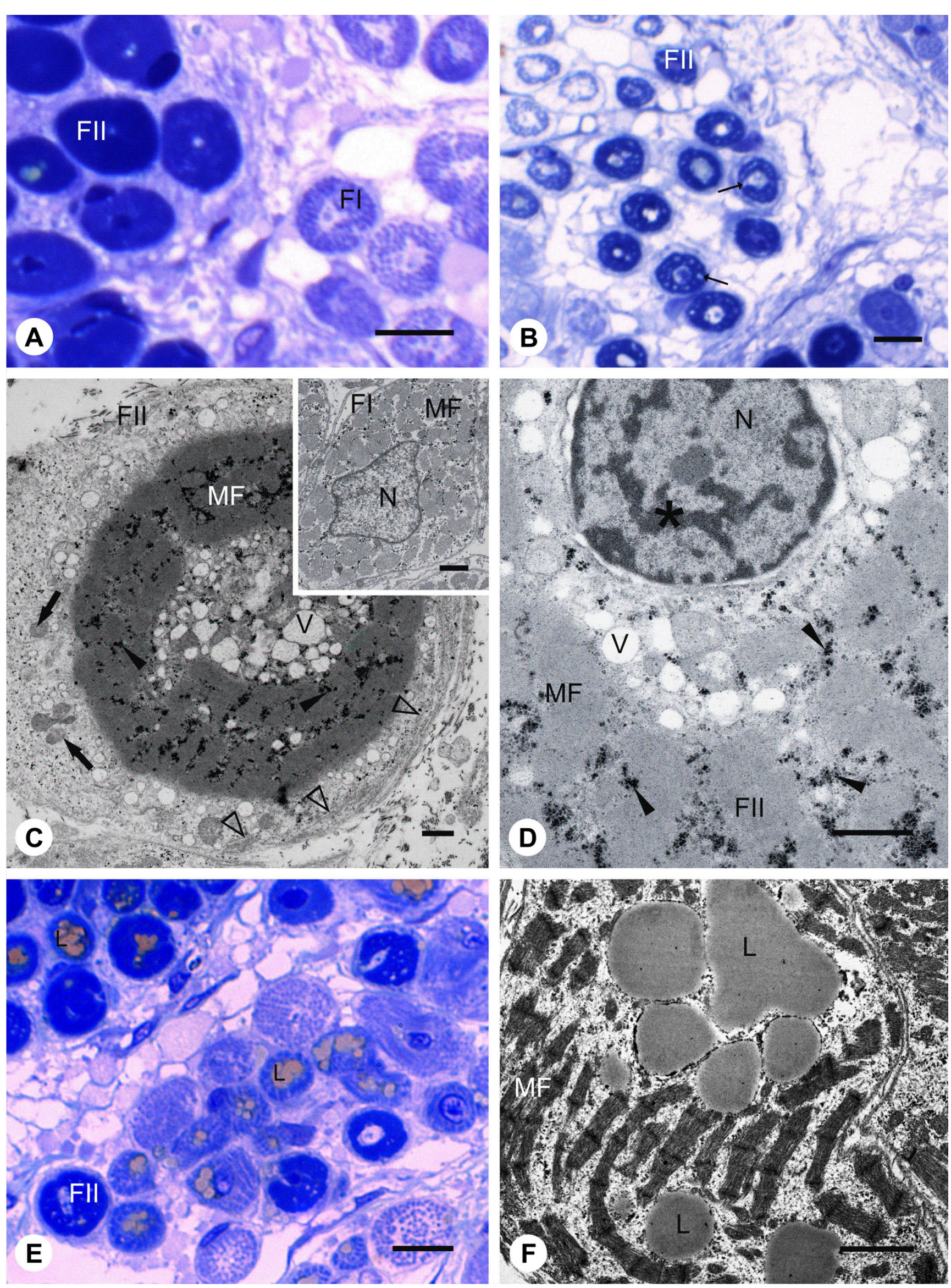
4 Fig. 2 Unique features of muscle differentiation in $N$. haje. a Stage 5: Cross-sections through the posterior trunk myotome. Heterogeneous population of muscle fibres in the myotome. First class of muscle fibres $(F I)$, second class of muscle fibres $(F I I)$. Semi-thin section, methylene blue staining, scale bar: $10 \mu \mathrm{m}$. b Stage 5: Cross-sections through the posterior trunk myotome. Class II of muscle fibres (FII), vesicles (black arrows). Semi-thin section, methylene blue staining, scale bar: $10 \mu \mathrm{m}$. c Stage 5: Cross-sections through the posterior trunk myotome. Ultrastructure of class II of muscle fibres (FII). Myofibrils $(M F)$, vesicles $(V)$, glycogen granules (black arrowheads), mitochondria (black arrows), endoplasmic reticulum (empty arrowheads). Inset: Ultrastructure of class I muscle fibres $(F I)$. Myofibrils $(M F)$, nucleus $(N)$. TEM, scale bar: $1 \mu \mathrm{m}$. d Stage 5: Cross-sections through the posterior trunk myotome. Ultrastructure of class II of muscle fibres $(F I I)$. Myofibrils $(M F)$ nucleus $(N)$ rich in heterochromatin (asterisk), vesicles $(V)$, glycogen granules (black arrowheads). TEM, scale bar: $1 \mu \mathrm{m}$. e Stage 6: Cross-sections through the posterior trunk myotome. Numerous lipid droplets $(L)$ in sarcoplasm of class II of muscle fibres (FII). Semi-thin section, methylene blue staining, scale bar: $10 \mu \mathrm{m}$. f Stage 6: Longitudinal through the posterior trunk myotome. Ultrastructure of class II of muscle fibres. Numerous lipid droplets $(L)$ in the sarcoplasm, myofibrils $(M F)$. TEM, scale bar: $1 \mu \mathrm{m}$

cycle. El-Deib (2005) described the lipid changes in blood serum and tissues of the Egyptian cobra. Applying physiological methods, the author determined lipid levels during different phases of the hibernation cycle (fatty acids, triglycerides, phospholipids and total cholesterol) in blood serum, liver, brain and cardiac and skeletal muscles. Data obtained from the study demonstrated that accumulation of lipids in the prehibernation phase is significantly high. During hibernation, the value of lipids significantly decreases, which suggests the consumption of lipids. Our results showed that during myogenesis in $N$. haje, there are some muscles that are capable of storing lipid droplets as the most economical form of storing energy. These muscles are also characterized by specific morphology (tightly packed myofibrils, large rim of sarcoplasm free from contractile apparatus and lipid droplets). Based on these morphological features, we believe that muscles capable of lipid storage belong to red/slow muscle fibres. The main part of the myotome in studied species is occupied by white/fast muscle fibres. These data strongly suggested that presence of lipid droplets in the sarcoplasm of some muscles during myogenesis might be a crucial adaptive mechanism for subsequent hibernation in adults.

\section{Perspectives}

Based on biology, reptiles are unique among vertebrate taxa. Snakes, closely related to lizards, are an extremely diverse group of reptiles. Studies on Egyptian cobra myotomal muscle differentiation and growth revealed similarities and differences compared to myogenesis described in amniotes including lizards. Based on our research, we revealed that the muscle fibre differentiation in studied species shares features with lizards, e.g. myoblasts fusion leads to multinucleated primary myotubes formation, and muscle fibre growth is accompanied by mononucleated cells whereas differences are connected with the presence of two classes of muscle fibres. In $N$. haje, the first class of muscle fibres represent typical muscle fibres morphology while the second class is characterized by sarcoplasmic lipid droplets surrounded by glycogen granules, the feature never observed during L. agilis myogenesis (Daczewska, unpublished data).

Our research for the first time revealed that the pattern of muscle differentiation depends on specific features of Egyptian cobra biology. The results of the present study have provided some answers to questions about the mechanisms of muscle differentiation and growth in the Egyptian cobra. However, many questions remain unanswered. Studies on muscle differentiation in reptiles are incomplete and require more detailed investigation, e.g. to find the origin of muscle progenitor cells in snakes and to define regulatory factors that control muscle fibre differentiation. The main question is: In what manner may the adaptation to different environmental conditions influence the mode of myogenesis? We believe that data obtained from Egyptian cobra myogenesis provide the basis for further reptilian investigation.

Acknowledgments The authors thank Sylwia Nowak form Laboratory of Microscopic Techniques (Faculty of Biological Sciences, Wroclaw University) and Dr. Danuta Urbańska-Jasik from the Department of Animal Histology and Embryology, University of Silesia for the technical assistance. We acknowledge the support of the Polish State Committee for Scientific Research, projects no. 1018/S/IZ/2014 and PSP/1S-0113001-1-01-06/2014.

Conflict of interest The authors declare that they have no competing interests.

Open Access This article is distributed under the terms of the Creative Commons Attribution 4.0 International License (http:// creativecommons.org/licenses/by/4.0/), which permits unrestricted use, distribution, and reproduction in any medium, provided you give appropriate credit to the original author(s) and the source, provide a link to the Creative Commons license, and indicate if changes were made.

\section{References}

Aerts P, Van Damme R, Vanhooydonck B, Zaaf A, Herrel A (2000) Lizard locomotion: how morphology meets ecology. Neth J Zool 50:261-277

Aubret F (2004) Aquatic locomotion and behaviour in two disjunct populations of Western Australian tiger snakes, Notechis ater occidentalis. Aust J Zool 52:357-368

Bentzinger CF, Wang YX, Rudnicki MA (2012) Building muscle: molecular regulation of myogenesis. Cold Spring Harb Perspect Biol 4: a008342

Brown M (1987) Change in fibre size, not number, in ageing skeletal muscle. Age Ageing 16:244-248

Buckingham M, Realaix F (2007) The role of Pax genes in the development of tissues and organs: Pax 3 and Pax 7 regulate muscle progenitor cells functions. Annu Rev Cell Dev Biol 23:645-673 
Daczewska M (2001) Mechanism of multinucleate myotomal muscle fibre formation in Hymenochirus boettgeri (Anura, Pipidae). Zoomorphology 121:27-36

Daughters RS, Chen Y, Slack JMW (2011) Origin of muscle satellite cells in the Xenopus embryo. Development 138:821-830

El-Deib S (2005) Lipid changes in blood serum and tissues of Egyptian Cobra Naja haje haje during the hibernation cycle. J Therm Biol 30: $51-59$

Fiedler I, Rehfeld C, Ender K, Henning M (1998) Histophysiological features of skeletal muscle and adrenal glands in wild-type and domestic pigs during growth. Archi Tierzuch 41:489-496

Fowler SP, Campion DR, Marks HL, Reagan JO (1980) An analysis of skeletal muscle response to selection for rapid growth in quail (Coturnix coturnix japonica). Growth 44:235-252

Garland TJ, Losos JB (1994) Ecological morphology of locomotor performance in squamate reptiles. In: Wainwright PC, Reilly SM (eds) Ecological morphology: integrative organismal biology. University of Chicago Press, Chicago, pp 240-302

Gasc JP (1981) Axial musculature. In: Gaus C, Parsons TS (eds) Biology of the reptilia vol. 11. Academic, New York, pp 355-435

Gleeson TT, Putnam RW, Bannett AF (1980) Histochemical, enzymatic and contractile properties of skeletal muscle fibres in the lizard Dipsosaurus dorsalis. J Exp Zool 214:293-302

Guthe KF (1981) Reptilian muscle: fine structure and physiological parameters. In: Gans C, Parsons TS (eds) Biology of the reptilia, vol. 11. Academic, New York, pp 265-354

Horst D, Ustanina S, Sergi C, Mikuz G, Juergens H, Braun T, Vorboyov E (2006) Comparative expression analysis of Pax3 and Pax7 during mouse myogenesis. Int J Dev Biol 50:47-54

Irshick DJ, Garland TJ (2001) Integrating function and ecology in studies of adaptation: investigation of locomotor capacity as a model system. Annu Rev Ecol Syst 32:367-396

Jayne BC (1982) Comparative morphology of the semispinalis-spinalis muscle of snakes and correlation with locomotion and constriction. J Morph 172:83-96

Kacperczyk A, Daczewska M (2006) Mixed mesodermal-mesenchymal origin of myotomal muscles in pike (Esox lucius: Telostei). Anat Histol Embryol 35:57-65

Kacperczyk A, Daczewska M (2008) The Australian lungfish (Neoceratodus forsteri)-fish or amphibian pattern of muscle development? Int J Dev Biol 52:279-286

Kacperczyk A, Jagla T, Daczewska M (2009) Pax-3 and Pax-7 label muscle progenitor cells during myotomal myogenesis in Coregonus lavaretus (Teleostei: Coregonidae). Anat Histol Embryol 38:411-418

Kacperczyk A, Jędrzejowska I, Daczewska M (2011) Differentiation and growth of myotomal muscles in a non-model tropical fish Pterophyllum scalare (Teleostei: Cichlidae). Anat Histol Embryol 40:411-418

Kalderon N, Gilula NB (1979) Membrane events involved in myoblasts fusion. J Cell Biol 81:411-425

Khannoon ER, Evans SE (2014) The embryonic development of the Egyptian cobra Naja h. haje (Squamata: Serpentes: Elapidae). Acta Zool 95:472-483

Kiełbówna L, Jedrzejowska I (2012) How is myogenesis initiated in Chordates? Folia Biol (Krakow) 60:107-119

Kusumi K, May CM, Eckalbar WL (2013) A large-scale view of the evolution of amniote development: insights from somitogenesis in reptiles. Curr Opin Genet Dev 23:491-497

Luft JH (1961) Improvement in epoxy resin embedding methods. J Biophys Biochem Cytol 9:409-414

McElroy EJ, Reilly SM (2009) The relationship between limb morphology, kinematics and force during running: the evolution of locomotor dynamics in lizards. Biol J Linn Soc 97:634-651

Merkel M (1995) Somitogenesis and myotomal myogenesis in European grayling Thymallus thymallus L. (Telostei). Zool Pol 40:119-130
Moritz S, Schilling N (2013) Fiber-type composition in the perivertebral musculature of lizards: implications for the evolution of the Diapsid trunk muscles. J Morph 274:294-306

Mosauer W (1935) The myology of the trunk region of snakes and its significance for ophidian taxonomy and phylogeny. Publ Univ Cal Los Angeles Biol Sci 1:81-121

Nagashima H, Uchida K, Yamamoto K, Kuraku S, Usuda R, Kuratani S (2005) Turtle-chicken chimera: an experimental approach to understanding evolutionary innovation in the turtle. Dev Dyn 232:149161

Nimmo MA, Snow RH (1983) The effect of ageing on skeletal muscle fibre characteristic in two inbred strains of mice. J Physiol 40:24-25

Olguin HC, Olwin BB (2004) Pax-7 up-regulation inhibits myogenesis and cell cycle progression in satellite cells: a potential mechanism for self-renewal. Dev Biol 275:375-388

Rehfeldt C, Fiedler I (1984) Postnatale Entwicklung der Muskelfasern im wachsenden Skelettmuskel der Labormaus. Arch Exp Veterinarmed $38: 178-192$

Reynolds ES (1963) The use of lead citrate at high $\mathrm{pH}$ as an electronopaque stain in electron microscopy. J Cell Biol 17:208-212

Rosenblatt JD, Woods RI (1992) Hypertrophy of rat extensor digitorum longus muscle injected with bupivacaine. a sequential histochemical, immunohistochemical, histological and morphometric study. J Anat 181:11-27

Rossi G, Messina G (2014) Comparative myogenesis in teleosts and mammals. Cell Mol Life Sci 71:3081-3099

Rowe RWE, Goldspink G (1969) Muscle fibre growth in five different muscle in both sexes of mice. J Anat 104:519-530

Rupik W (2002) Early development of the adrenal glands in the grass snake Natrix natrix L.(Lepidosauria, Serpentes). Adv Anat Embryol Cell Biol 164:1-102

Rupik W (2011) Structural and ultrastructural differentiation of the thyroid gland during embryogenesis in the grass snakeNatrix natrix $L$. (Lepidosauria, Serpentes). Zoology 114:284-297

Rupik W (2012) Hollowing or cavitation during follicular lumen formation in the differentiating thyroid of grass snake Natrix natrix L. (Lepidosauria, Serpentes) embryos? an ultrastructural study. Zoology 115:389-397

Rupik W (2013) Ultrastructural studies of cilia formation during thyroid gland differentiation in grass snake embryos. Micron 44:228-237

Rupik W, Swadźba E, Dubińska-Magiera M, Jędrzejowska I, Daczewska M (2012) Reptilian myotomal myogenesis-lessons from sand lizard Lacerta agilis L. (Reptilia, Lacertidae). Zoology 115:330-338

Schadereit R, Klein M, Rehfeldt C, Kreienbring F, Krawietzki K (1995) Influence of nutrient restriction and realimentation on protein energy metabolism, organ weights and muscle structure in growing rats. J Anim Physiol Anim Nutr (Berl) 74:253-268

Schleich HH, Kästle W, Kabisch K (1996) Amphibians and reptiles of North Africa: biology, systematics, field guide. Koeltz Scientific Book, Königstein, pp 1-625

Seale P, Sabourin LA, Girgis-Gabardo A, Mansouri A, Gruss P, Rudnicki MA (2000) Pax7 is required for the specification of myogenic satellite cells. Cell 102:777-786

Siegiel AL, Gurevich DB, Currie PD (2013) A myogenic precursor cell that could contribute to regeneration in zebrafish and its similarity to the satellite cell. FEBS J 280:4074-4088

Summers PJ, Medrano JF (1994) Morphometric analysis of skeletal muscle growth in the high growth mouse. Growth Dev Aging 58:135148

Swadźba E, Rupik W (2010) Ultrastructural studies of epidermis keratinization in grass snake embryos Natrix natrix L. (Lepidosauria, Serpentes) during late embryogenesis. Zoology 113:339-360

Swadźba E, Rupik W (2012) Cross-immunoreactivity between the LH1 antibody and cytokeratin epitopes in the differentiating epidermis of embryos of the grass snakeNatrix natrix L. during the end stages of embryogenesis. Protoplasma 249:31-42 
Swatland HJ (1975) Myofibre number and myofibrillar development in neonatal pig. Zentralbl Veterinarmed A 22:756-764

Yin H, Price F, Rudnicki MA (2013) Satellite cells and muscle stem cells niche. Physiol Rev 93:23-67
Zammit PS, Relaix F, Nagata Y, Perez Riuz A, Collins CA, Partridge TA, Beauchamp JR (2006) Pax7 and myogenic progression in skeletal muscle satellite cells. J Cell Sci 119: $1824-1832$ 